\title{
Power saving through state retention in IGZO-TFT AMOLED displays for wearable applications
}

\author{
Soeren Steudel ${ }^{a}$, Jan-Laurens P.J. van der Steen ${ }^{c}$, Manoj Nag ${ }^{a b}$, Tung Huei \\ $\mathrm{Ke}^{\mathrm{a}}$, Steve Smout ${ }^{\mathrm{a}}$, Thijs Bel ${ }^{\mathrm{c}}$, Karin van Diesen ${ }^{\mathrm{c}}$, Gerard de Haas ${ }^{\mathrm{c}}$, Joris \\ Maas c , Joris de Riet c, Madelon Rovers c, Roy Verbeek c, Yen-Yu Huang ${ }^{\text {, }}$, \\ Shin-Chuan Chiang ${ }^{d}$, Marc Ameys ${ }^{a}$, Florian De Roose ${ }^{a b}$, Wim Dehaene ${ }^{a b}$, Jan \\ Genoe $^{\mathrm{ab}}$, Paul Heremans ${ }^{\mathrm{ab}}$, Gerwin Gelinck ${ }^{\mathrm{c}}$, Auke Jisk Kronemeijer ${ }^{\mathrm{c}}$ \\ a IMEC, Kapeldreef 75, 3001 Leuven, Belgium \\ ${ }^{b}$ ESAT, KU Leuven, Kasteelpark Arenberg 10, 3001, Leuven, Belgium \\ c TNO/Holst Centre, High Tech Campus 31, 5656 AE Eindhoven, The Netherlands \\ ${ }^{d}$ Chungwha Picture Tubes, LDT. 1127 Heping Rd., Bade City, Taoyuan, Taiwan, 334, R.O.C.
}

\begin{abstract}
We present a $q H D(960 \times 540$ with 3 sub-pixels) top-emitting AMOLED display with 340ppi resolution using a self-aligned (SA) IGZO TFT backplane on polyimide-foil with humidity barrier. The back plane process flow is based on a 7 layer photolithography process with a $C D=4 u m$. We implement a $2 T 1 C$ pixel engine and use commercial source driver IC made for LTPS. By using IGZO TFT and leveraging the extremely low off-current, we can switch-off the power to the source and gate driver while maintaining the image un-changed for several minutes. We demonstrate that, depending on the image content, low-refresh operation yields reduction in power consumption of up to $50 \%$ compared to normal (continuous) operation. We show that with further increase in resolution, the power saving through state retention will be even more significant.
\end{abstract}

Keywords: flexible displays, AMOLED, metal-oxide semiconductors, self-aligned TFT, state retention

\section{INTRODUCTION}

Market research predicts [1] that by 2020 , more than $70 \%$ of shipped smartphone displays will be AMOLED with flexible or curved AMOLED displays accounting for more than half of the overall smartphone and wearable display market. Resolution, mechanical flexibility and power consumption are hereby the main drivers for further development of AMOLED displays. Especially for wearables like smart watches, power consumption is a decisive argument for the choice of display technology.

A popular approach to lower the power consumption are reflective LCD displays with memory-in-pixel (MIP). Depending on the light conditions and display content, a backlight can be switched-on and the display runs with video refresh rate. Otherwise, if the content does not require constant refresh, the refresh rate can be slowed down or completely stopped while the image is retained although depending on the implementation with limited grey levels. In case of AMOLED technology, slowing down the frame rate or MIP [3] are less obvious energy saving strategies since the power required for light emission is generally dominant compared to the refreshing of the frame. This work attempts to quantify and measure the energy saving enabled by state retention based on the extremely low loff current of IGZO TFT in AMOLED displays.

\section{DISPLAY FABRICATION}

Our TFT backplane [3] is based on self-aligned IGZO TFT. The choice of IGZO TFT allows a monolithic integration whereby the TFT is directly processed on a flexible thin-film moisture barrier [4]. Only at the end of the full process flow, the display is released from the temporary glass substrate by laser or mechanical delamination.

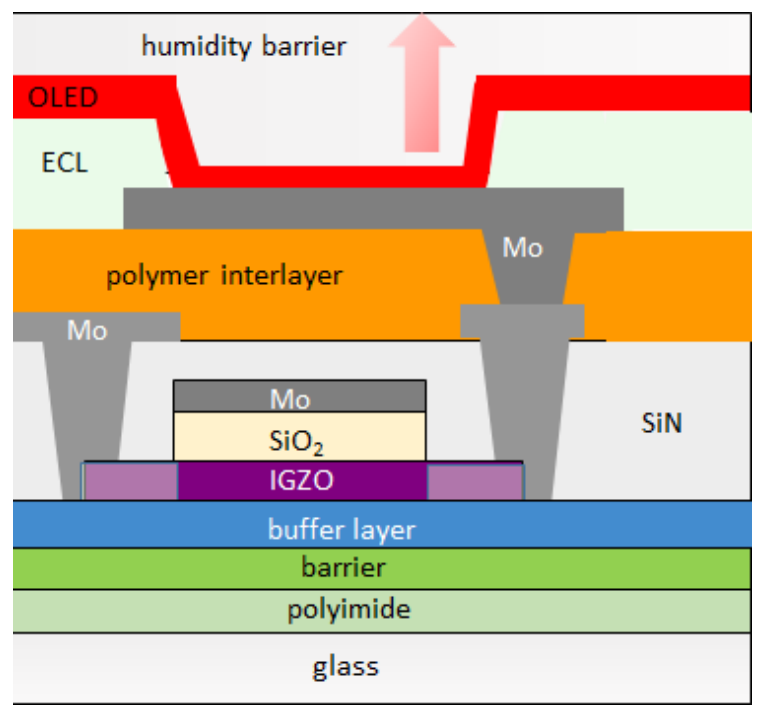

Fig.1. Cross-sectional view of the seven mask backplane of the AMOLED display 
Using a SA architecture allows to realize a TFT backplane with only 4 mask layers and reduced parasitic overlap capacitance (and therefore RC delay) compared to either ESL (etch-stop-layer) or BCE (back-channel-etch) architecture. This is especially beneficial for larger displays like $4 \mathrm{k} 2 \mathrm{k}$ with higher frame rates and integrated line driver. The overall display integration scheme of frontplane and backplane is shown in Figure 1.

On a temporary Gen1 (320mm x 350mm) glass carrier with a 16um thick solution coated polyimide film, a humidity and oxygen barrier [5] is deposited followed by a buffer layer to create an improved interface to the IGZO. Afterwards, IGZO (metal ratio $=1: 1: 1$ ) is sputtered by DC-PVD followed by a wet-etch step to define the active semiconductor area. In a further step we deposit 200nm PECVD $\mathrm{SiO}_{2}$ as a gate dielectric. Next, we deposit 100nm Mo-alloy as gate-metal. The gate/dielectric stack is patterned within the same masking step. Subsequently we deposit 200nm CVD SiN. The CVD SiN fulfills the double purpose of intermetal dielectric and doping the IGZO in the areas not covered by the gate/dielectric stack with hydrogen. The contact opening (CO) for the Source-Drain (SD) contacts are opened up by dry etching and $100 \mathrm{~nm}$ Mo is deposited and patterned to define the SD-contacts. The last step in the TFT process is a final anneal. Afterwards we deposit and pattern an interlayer dielectric, metal anode and an edge cover dielectric layer (ECL). This is followed by the deposition of an inverted orange small molecule OLED-stack (7cd/A), a transparent cathode and a transparent thin film barrier. For the purpose of verifying our stack integrity, backplane functionality and power consumption, we use monochrome smOLEDs instead of full RGB, despite the 3 sub-pixel design of the backplane, which is fully compatible with an RGB frontplane process.

The design rules for all layers are fixed to a $C D=4 \mu \mathrm{m}$ and an overlay $<2 \mu \mathrm{m}$, compatible with an exposure using a large GenX scanner. We target a drive current of $0.5 \mu \mathrm{A} @$ $\mathrm{V}_{\mathrm{DS}}=8 \mathrm{~V}$. A conventional $2 \mathrm{~T} 1 \mathrm{C}$ pixel scheme has been implemented, employing a drive TFT of $\mathrm{W} / \mathrm{L}=10.5 / 7.5 \mu \mathrm{m} / \mu \mathrm{m}$ and select TFT of $\mathrm{W} / \mathrm{L}=10 \mu \mathrm{m} / 4 \mu \mathrm{m}$. The longer channel length of the drive TFT improves the output resistance to $>64 \mathrm{M} \Omega$.

For a channel length of $5 \mu \mathrm{m}$, we achieve a field effect mobility of $\sim 12 \mathrm{~cm}^{2} / \mathrm{Vs}$. The transfer characteristics and a picture of the realized TFT is depicted in Figure 2 and the TFT values summarized in Table 1 . The loff shown in Figure 2 is in fact not the loff due to leakage but due to the limitations of the measurement setup used for mapping. The real loff is significantly below $<<1 \mathrm{e}-15 \mathrm{~A} / \mathrm{um}$ and can only be measured with a much higher W/L TFT.

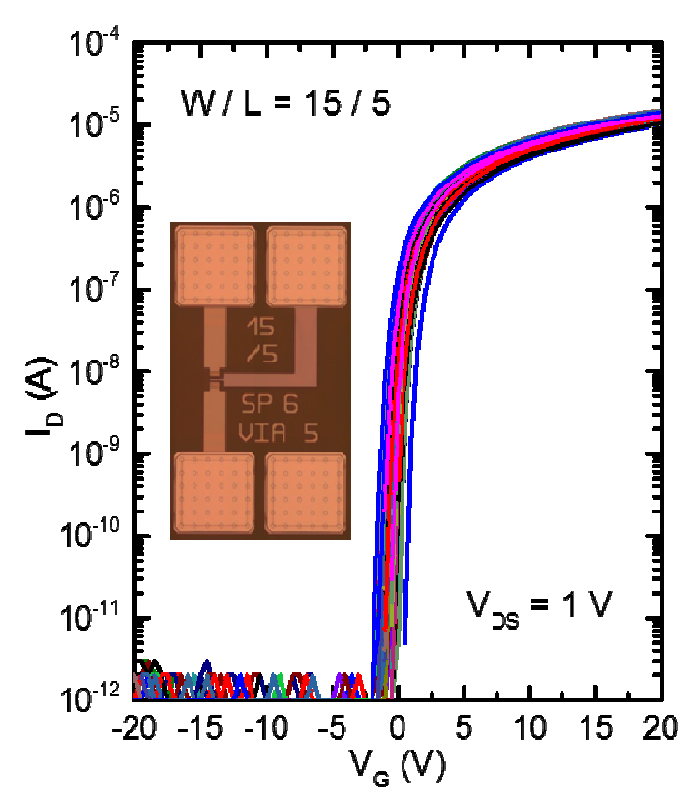

Fig.2. Transfer characteristics of $75 x$ SA-TFT with $\mathrm{W} / \mathrm{L}=15 \mu \mathrm{m} / 5 \mu \mathrm{m}$ across a Gen1 plate (inset) TFT image

Table 1. Summary of TFT parameter

\begin{tabular}{|c|c|c|c|c|}
\hline $\begin{array}{l}W / L \\
{[\mu m / \mu m]}\end{array}$ & $\begin{array}{l}\text { pFET } \\
{\left[\mathrm{cm}^{2} / \mathrm{Vs}\right]}\end{array}$ & $\begin{array}{l}\text { Von } \\
\text { [V] }\end{array}$ & $\begin{array}{l}\mathrm{s}^{-1} \\
{[\mathrm{dec} / \mathrm{V}]}\end{array}$ & $\begin{array}{l}\text { Rout } \\
\text { [M } \Omega]\end{array}$ \\
\hline $10.5 / 7.5$ & 11.3 & -0.8 & 0.3 & 64 \\
\hline $9 / 4$ & 11.5 & -1.1 & 0.3 & 33 \\
\hline
\end{tabular}

An picture of the 340ppi TFT backplane after the TFT process and after full display manufacturing can be seen in Figure 3.

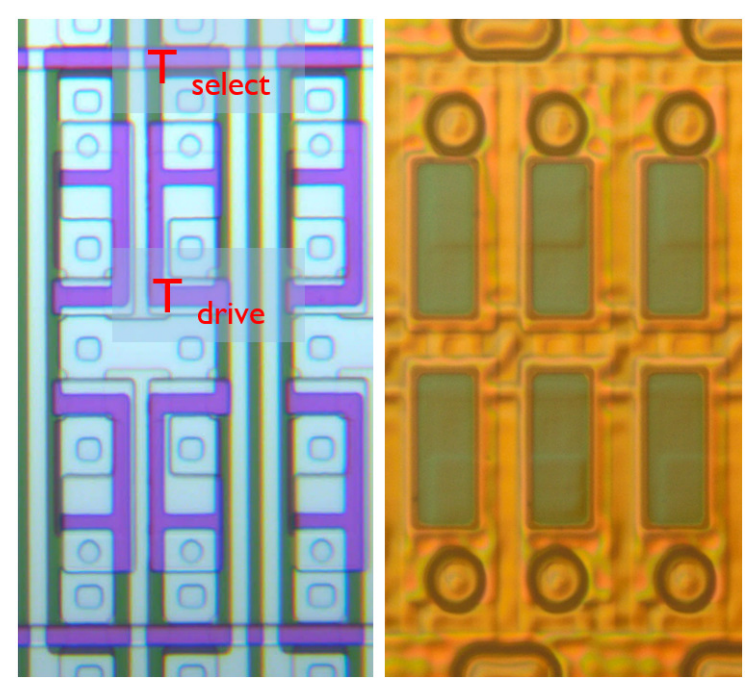

Fig.3 Microscopic picture of the pixel (left) after TFT process; (right) after full display process

Finally, to drive the display we bonded onto the PI substrate commercial COG gate driver IC with 480 channels and a LTPS source driver IC with 540 
channels. An in-panel 3:1 MUX allows to switch between the 3 sub-pixels.

The layout of the qHD $(960 \times 540 \times 3$ sub pixel $)$ display can be seen in Figure $4 \mathrm{a}$ and with an image applied in $4 \mathrm{~b}$.
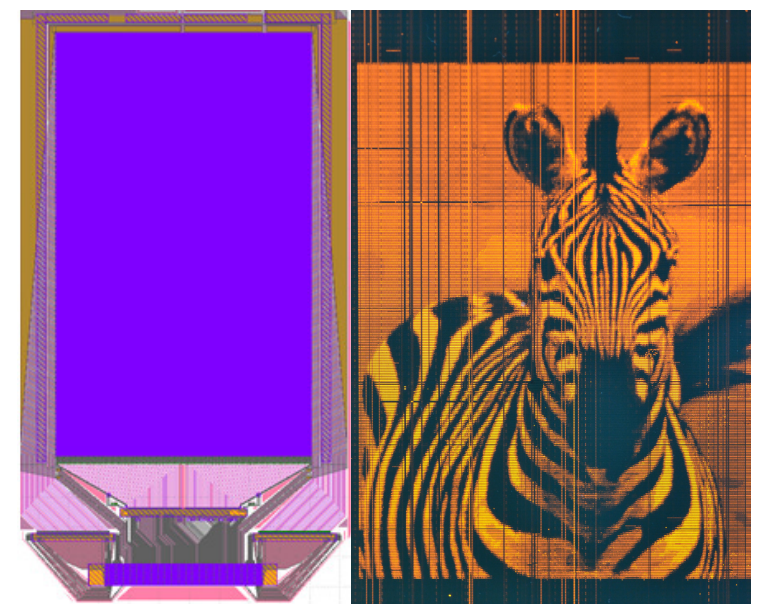

Fig.4. qHD AMOLED display (a) layout; (b) with applied image

At the end of the process flow, we release the AMOLED display by laser. The overall display is less than $60 \mu \mathrm{m}$ thick and can be bent with a radius of $5 \mathrm{~mm}$ as shown in Figure 5.

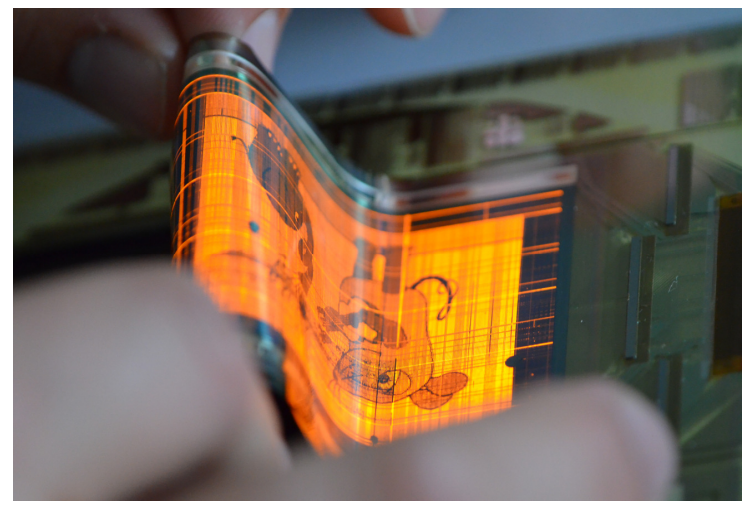

Fig.5. Released qHD AMOLED display

\section{DISPLAY CHARACTERIZATION}

The overal specifications of the display are listed in table 2.

Table 2. Display specification of this work

\begin{tabular}{|l|l|}
\hline Specification & Data \\
\hline Panel size & $3.2 "(4.2 \times 7.2 \mathrm{~mm})$ \\
\hline Pixel & qHD $(960 \times 540 \times 3)$ \\
\hline Resolution & $340 \mathrm{ppi}$ \\
\hline RGB MUX & Yes \\
\hline Date driver & LTPS driver IC \\
\hline
\end{tabular}

\begin{tabular}{|l|l|}
\hline Bezel width & $5 \mathrm{~mm}($ no GIP) \\
\hline Pixel engine & $2 \mathrm{~T} 1 \mathrm{C}$ \\
\hline TFT & Self-aligned IGZO \\
\hline Frame rate & $60 \mathrm{~Hz}$ \\
\hline Thickness & $<60 \mathrm{um}$ \\
\hline Substrate & Polyimide \\
\hline
\end{tabular}

In the next step, we tested the state retention of the display by applying an image and switching-off the power supply to the gate and source driver IC completely while maintaining the supply voltage at the OLED.

As can be seen in Figure 6 , the images are retained unchanged over more than 300s. ( Note: There are a several lines with line defects that are already visible at $\mathrm{T}=0 \mathrm{~s}$ that are getting more pronounced at $\mathrm{T}=300 \mathrm{~s}$.)

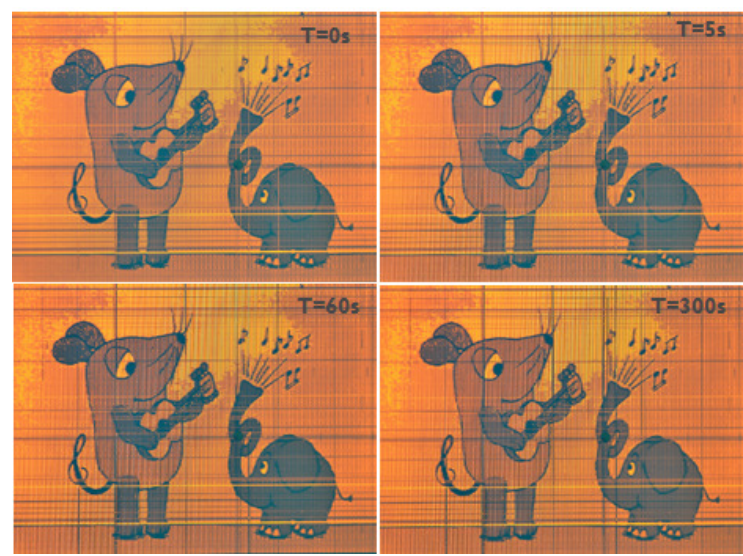

Figure 6. AMOLED display with applied image during standard driving $(\mathrm{T}=0 \mathrm{~s})$ and $5 \mathrm{~s}, 60 \mathrm{~s}$ and $300 \mathrm{~s}$ after the driver ICs have been switched off.

Subsequently, we measured the power consumption of the display. Hereby we can measure seperately the current through the logic of both the gate and source driver, the current required by the select high and low and the current through the OLED.

Applying a uniform white image ( fully-on ) with a frame rate of 30fps, a brightness of $\sim 100 \mathrm{~cd} / \mathrm{m}^{2}$ and a voltage across the OLED + drive TFT of $10 \mathrm{~V}$, we measured a power consumption of approximitly POLED $460 \mathrm{~mW}$. The power consumption of the source and gate driver IC was Pdriver_IC $41 \mathrm{~mW}$. Applying a white or black imagechanges the power consumption of the logic by only $1-2 \mathrm{~mW}$. The power consumption of the select high and low remained below $P_{\text {select }}<1 \mathrm{~mW}$ and is therefore nearly negligible. We have a select line capacitance of approx. 24pF and a Vg,h=10V and Vg,l=-10V. Changing the frame rate or the $\mathrm{Vg}, \mathrm{h}$ and $\mathrm{Vg}$, I will influence this $P_{\text {select }}$ value by only factor $2-3$. In the measured case, switching-off the driver IC and relying on state retention, we can save for a fully-on image $\sim 8 \%$ of the energy. 
Depending on different usage scenarios and settings, the potential power saving can vary widely. The biggest effect stems from the target brightness and the actually shown content. If a newspaper article ( black text on white background ) is shown with a brightness of $500 \mathrm{~cd} / \mathrm{m}^{2}$ to be read in bright daylight, the power consumption of the driver IC becomes irrelevant. However if we only present some basic information on a smart watch, e.g. the time and status information during sleep mode ( e.g. Always On Display in Samsung Galaxy Note 4 Edge ), the possibility to completly switch-off the display driver IC enabled by the low loff of the IGZO TFT, becomes a significant energy saver.

In table 3, we show estimates for diffrerent usage scenarios assuming a brightness of $100 \mathrm{~cd} / \mathrm{m}^{2}$ with an average efficency of RGB OLED @ 10cd/A. In a sleep information mode ( $10 \%$ of OLED pixels on), the energy saving can be more than $50 \%$. If we increase the pixel density while maintaing the same size of the display, the potential energy saving increases further. The power consumption of the OLED remains constant with constant display area, however the number of driver IC or the number of controlled I/O per IC increases. The time required to switch the driver $\mathrm{IC}$ on and refresh the frame is $<30 \mathrm{~ms}$. This means that, even if the applied image changes every second ( e.g. analogue watch), implementing state retention can lead to energy savings.

Table 3. Estimated power saving for AMOLED displays using IGZO-TFT state retention

\begin{tabular}{|c|c|c|c|c|c|}
\hline $\begin{array}{c}\text { Resolution } \\
\text { [ppi] }\end{array}$ & \multicolumn{2}{|c|}{$\begin{array}{c}\text { Power [mW] } \\
\text { OLED + drive TFT }\end{array}$} & $\begin{array}{c}\text { Power [mW] } \\
\text { IC }\end{array}$ & \multicolumn{2}{|c|}{ Power saving } \\
\hline Size 3.2" & $\begin{array}{c}100 \% \\
\text { on }\end{array}$ & $\begin{array}{c}10 \% \\
\text { on }\end{array}$ & $\begin{array}{c}\text { (line select + } \\
\text { data driver ) }\end{array}$ & $\begin{array}{c}100 \% \\
\text { on }\end{array}$ & $\begin{array}{c}10 \% \\
\text { on }\end{array}$ \\
\hline $340 @$ qHD & 300 & 30 & 40 & $12 \%$ & $57 \%$ \\
\hline 680 @ FHD & 300 & 30 & 80 & $21 \%$ & $72 \%$ \\
\hline $1360 @ 4 k 2 k$ & 300 & 30 & 160 & $35 \%$ & $84 \%$ \\
\hline
\end{tabular}

\section{CONCLUSION}

A 340ppi flexible AMOLED display with IGZO TFT has been shown. Utilizing the very low loff of IGZO TFT, we implemented state retention enabling us to completely switch-off the driver IC for several hundred seconds while maintaining the image. We measured the resulting power saving and extrapolated those results for different usage scenarios.

This work was carried out in the frame of the Holst Centre, a joint collaboration between IMEC and TNO.

\section{REFERENCES}

[1] UBI Research, 2016 OLED Display Annual Report

[2] Y. Huang, B. Hekmatshoar, S. Wagner and J. C. Sturm, "High retention-time nonvolatile amorphous silicon TFT memory for static active matrix OLED display without pixel refresh," 68th Device Research Conference, South Bend, IN, 2010, pp. 179-180.

[3] S. Steudel et al, Flexible AMOLED display with integrated gate driver operating at operation speed compatible to $4 k 2 k$, SID 15 DIGEST, pp. 427-430

[5] Li, FM et al. Flexible Barrier Technology for Enabling Rollable AMOLED Displays and Upscaling Flexible OLED Lighting, SID 13 DIGEST, pp. 199-202 